\title{
Die Riemannsche Vermutung
}

\author{
Jürg Kramer
}

\section{Einführung}

In dem hier vorzustellenden Millenniumsproblem handelt es sich um eine zahlentheoretische Fragestellung aus dem 19. Jahrhundert, die seit ein paar Jahren auch überraschende Zusammenhänge zu anderen Gebieten der Mathematik und der theoretischen Physik erkennen lässt. Die Problemstellung hat ihren Ursprung bei der Frage nach der Dichte der Primzahlen im Bereich der natürlichen Zahlen. Um den Leser in den Problemkreis einzuführen, wollen wir einfach beginnen. Wir bezeichnen mit $\mathbb{N}$ die Menge der natürlichen Zahlen, d.h.

$$
\mathbb{N}=\{0,1,2,3, \ldots\} .
$$

Mit $\mathbb{P}$ bezeichnen wir die Menge der Primzahlen, d.h. die Menge aller natürlichen Zahlen grösser als Eins, die nur durch sich selbst und durch Eins teilbar sind, also

$$
\mathbb{P}=\{2,3,5,7,11,13, \ldots, 229, \ldots\} .
$$

Wir erinnern nun an zwei Tatsachen, die vermutlich den meisten Lesern wohl bekannt sind.

Das erste ist die Tatsache, dass jede positive natürliche Zahl $n$ sich bis auf die Reihenfolge eindeutig als Produkt von Primzahlpotenzen darstellen lässt, d.h. es gibt Primzahlen $p_{1}, \ldots, p_{r}$ und natürliche Zahlen $\alpha_{1}, \ldots, \alpha_{r}$ derart, dass die Gleichheit

$$
n=p_{1}^{\alpha_{1}} \cdot \ldots \cdot p_{r}^{\alpha_{r}}
$$

besteht. Dies ist der Inhalt des sogenannten Fundamentalsatzes der Zahlentheorie, der mit anderen Worten besagt, dass die Primzahlen die multiplikativen Bausteine der natürlichen Zahlen sind.

Als zweites erinnern wir an den Satz von Euklid, dass es nämlich unendlich viele Primzahlen gibt. Dies sieht man leicht wie folgt ein: Man nimmt im Gegenteil zur Behauptung an, dass es nur endlich viele Primzahlen $p_{1}, \ldots, p_{N}$ gibt. Damit bildet man die (sehr grosse) Zahl

$$
m=p_{1} \cdot \ldots \cdot p_{N}+1 \text {. }
$$

Nun besitzt die natürliche Zahl $m$ einerseits mindestens einen Primteiler $q$. Da die Zahl $m$ andererseits bei Division durch jede der Primzahlen $p_{1}, \ldots, p_{N}$ den Rest 1 lässt, muss 
$q \neq p_{j}(j=1, \ldots, N)$ gelten. Dies ist ein Widerspruch zu unserer Annahme und beweist somit die Unendlichkeit der Anzahl der Primzahlen.

Nach dem Satz von Euklid gibt es also beliebig grosse Primzahlen. Dieser Umstand spielt heute in der Kryptographie eine wichtige Rolle. Die gegenwärtig grösste bekannte Primzahl der Form $p=2^{n}-1$, eine sogenannte Mersennesche Primzahl, lautet

$$
p=2^{13466917}-1 ;
$$

sie hat mehr als 4 Mio. Stellen (siehe [10]). Würde man alle Stellen ausdrucken, so wären bei der hier gewählten Schriftgrösse mehr als 1000 A4-Seiten notwendig.

\section{Die Riemannsche Zetafunktion}

Für reelles oder allgemeiner komplexes $s=\sigma+i t$ betrachten wir die Reihe

$$
\sum_{n=1}^{\infty} \frac{1}{n^{s}}=1+\frac{1}{2^{s}}+\frac{1}{3^{s}}+\frac{1}{4^{s}}+\frac{1}{5^{s}}+\ldots
$$

Für $t=0$ und $\sigma>1$ erkennen wir die aus den Grundvorlesungen der Analysis wohlbekannte konvergente Reihe, die oft als Majorante herangezogen wird; für $t=0$ und $\sigma=1$ erhalten wir die harmonische Reihe, welche bekanntlich divergiert, allerdings sehr langsam. Betrachtet man nun die Reihe (1) als Funktion von $s$, so lässt sich das folgende dazu festhalten: Die Reihe (1) konvergiert für $\operatorname{Re} s=\sigma>1$ absolut und lokal gleichmässig und definiert dort eine holomorphe Funktion, die Riemannsche Zetafunktion $\zeta(s)$.

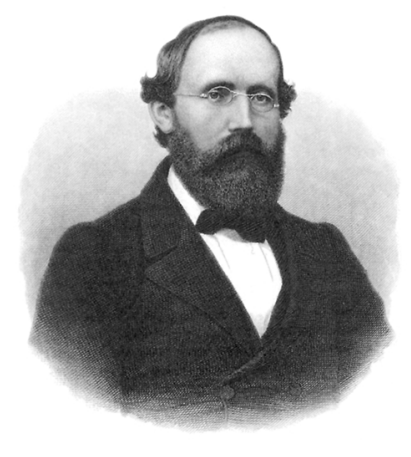

Fig. 1 Bernhard Riemann

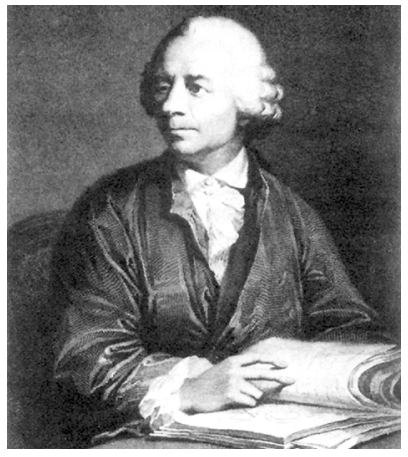

Fig. 2 Leonhard Euler

Mit Hilfe der Poissonschen Summationsformel beweist man weiter die Funktionalgleichung

$$
\pi^{-s / 2} \Gamma(s / 2) \zeta(s)=\pi^{-(1-s) / 2} \Gamma((1-s) / 2) \zeta(1-s) ;
$$

hierbei ist $\Gamma(s)$ die Eulersche Gammafunktion. Mit der Funktionalgleichung (2) zeigt man, dass sich $\zeta(s)$ zu einer meromorphen Funktion auf die gesamte komplexe Ebene $\mathbb{C}$ mit einem Pol erster Ordnung an der Stelle $s=1$ mit Residuum 1 fortsetzen lässt. 
Diese und weitere grundlegende Eigenschaften der Riemannschen Zetafunktion werden z.B. in den Lehrbüchern [2] oder [6] dargestellt.

Die hervorragende Bedeutung der Riemannschen Zetafunktion für die Arithmetik wird durch die beiden folgenden Resultate, die bereits durch L. Euler (1707-1783) entdeckt wurden, deutlich:

(i) Die Riemannsche Zetafunktion besitzt für $\operatorname{Re} s>1$ die sogenannte Eulersche Produktentwicklung, d.h.

$$
\zeta(s)=\prod_{p \in \mathbb{P}}\left(1-p^{-s}\right)^{-1}
$$

Die Gültigkeit dieser Produktentwicklung sieht man unmittelbar ein, indem man den Term $\left(1-p^{-s}\right)^{-1}$ durch die geometrische Reihe $\sum_{m=0}^{\infty} p^{-m s}$ ersetzt und dann sukzessive das Produkt über alle Primzahlen bildet. Dabei erkennt man, dass das Bestehen der Eulerschen Produktentwicklung für $\zeta(s)$ gleichbedeutend zum Fundamentalsatz der Arithmetik ist.

(ii) Als zweites erkennt man, dass der Satz von Euklid über die Unendlichkeit der Menge der Primzahlen äquivalent zur Tatsache ist, dass $\zeta(s)$ an der Stelle $s=1$ einen Pol hat. Mit Hilfe der Eulerschen Produktentwicklung ergibt sich nämlich für $s \downarrow 1$

$$
\lim _{s \downarrow 1} \zeta(s)=\infty
$$

d.h. das unendliche Produkt

$$
\prod_{p \in \mathbb{P}} \frac{1}{1-p^{-1}}
$$

divergiert, was die Unendlichkeit der Menge $\mathbb{P}$ zur Folge hat.

\section{Die Primzahlfunktion}

Da es also unendlich viele Primzahlen gibt, kann man versuchen, deren Dichte in der Menge der natürlichen Zahlen zu ermitteln. Dazu bezeichnen wir für positives, reelles $x$ mit $\pi(x)$ die Anzahl der Primzahlen, die kleiner oder gleich $x$ sind, d.h.

$$
\pi(x)=\#\{p \in \mathbb{P} \mid p \leq x\} .
$$

Dies definiert eine reellwertige Funktion, die sogenannte Primzahlfunktion. Für kleine Werte von $x$ erkennen wir $\pi(x)$ als Treppenfunktion (siehe Fig. 3); für grosse Werte von $x$ tritt der Treppenfunktionscharakter von $\pi(x)$ in den Hintergrund, und es scheint sich asymptotisch eine glatte Funktion zu zeigen (siehe Fig. 4). Dieses Phänomen ist im wesentlichen der Inhalt des Primzahlsatzes, der besagt, dass für $x \rightarrow \infty$ die Asymptotik

$$
\pi(x) \sim \frac{x}{\log x}
$$

besteht, welche mit Hilfe des Integrallogarithmus

$$
\operatorname{Li}(x)=\int_{2}^{\infty} \frac{\mathrm{d} t}{\log t}
$$


noch verbessert werden kann zu

$$
\pi(x) \sim \operatorname{Li}(x)
$$

(siehe Fig. 3, 4). Dieser Satz wurde bereits von C.F. Gauß (1777-1855) vermutet, aber erst im Jahr 1896 durch J. Hadamard (1865-1963) und C. de la Vallée Poussin (18661962) vollständig bewiesen.

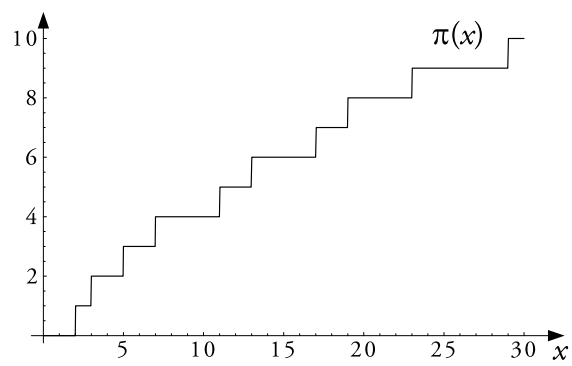

Fig. 3 Primzahlfunktion $\pi(x)$

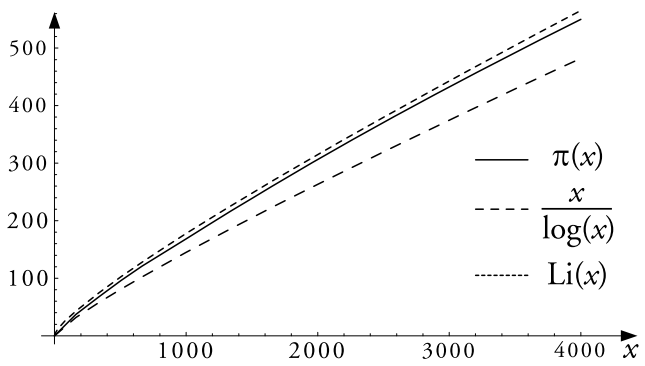

Fig. 4 Primzahlfunktion $\pi(x)$

Im Jahr 1949 fanden A. Selberg und P. Erdös (1913-1996) einen elementaren Beweis des Primzahlsatzes, der weder die Riemannsche Zetafunktion noch die Funktionentheorie verwendet (siehe [2], Chapter I). Vor kurzem, im Jahr 1997, hat D. Zagier basierend auf einer Idee von D.J. Newman einen sehr kurzen Beweis des Primzahlsatzes gegeben, der neben einigen sehr elementaren arithmetischen Tatsachen nur den Cauchyschen Integralsatz heranzieht (siehe [9]).

Nach dem Primzahlsatz besteht also für die Primzahlverteilung die Formel

$$
\pi(x)=\operatorname{Li}(x)+R(x)
$$

mit einem Restglied $R(x)$, welches

$$
\frac{R(x)}{\operatorname{Li}(x)} \underset{x \rightarrow \infty}{\longrightarrow} 0
$$

erfüllt. Nunmehr ist es natürlich von Interesse, das Restglied $R(x)$ in den Griff zu bekommen. Dies führt uns endlich zu dem in diesem Beitrag vorzustellenden Millenniumsproblem.

Riemannsche Vermutung: Diese Vermutung besagt, dass das Restglied $R(x)$ für $x \rightarrow \infty$ von der Grössenordnung

$$
R(x)=O(\sqrt{x} \log x)
$$

ist.

Bis heute ist man noch weit davon entfernt, diese Vermutung beweisen zu können. Noch immer ist E. Littlewoods (1885-1977) Abschätzung aus dem Jahre 1922 im wesentlichen unübertroffen. Seine Abschätzung für das Restglied $R(x)$ lautet

$$
R(x)=O\left(x \cdot e^{-C \sqrt{\log x \log \log x}}\right)
$$


mit einer positiven Konstanten C (siehe [2], Chapter III). Mit Hilfe der sogenannten „Expliziten Formeln“ der analytischen Zahlentheorie lässt sich die Riemannsche Vermutung als Vermutung über die Lage der Nullstellen von $\zeta(s)$ umformulieren. In dieser Form hat B. Riemann (1826-1866) seine Vermutung ursprünglich festgehalten.

Äquivalente Formulierung der Riemannschen Vermutung: Abgesehen von den sogenannten ,trivialen“ Nullstellen der Riemannschen Zetafunktion bei $s=-2,-4,-6$, $-8, \ldots$ befinden sich sämtliche weiteren Nullstellen von $\zeta(s)$ auf der kritischen Geraden $\{s \in \mathbb{C} \mid \operatorname{Re} s=1 / 2\}$.

Man findet die Original-Formulierung von Riemanns Vermutung in seinem Beitrag „Ueber die Anzahl der Primzahlen unter einer gegebenen Grösse“ in den Monatsberichten der Berliner Akademie vom November 1859 (siehe [8], p. 180). Nach Einführung der Funktion

$$
\xi(t)=\pi^{-s(t) / 2} \Gamma(s(t) / 2)(s(t)-1) \zeta(s(t))
$$

mit $s(t)=1 / 2+i t$ bemerkt Riemann dort: „Man findet nun in der That etwa soviele reelle Wurzeln innerhalb dieser Grenzen, und es ist sehr wahrscheinlich, dass alle Wurzeln reell sind.“

\section{Beweisansätze}

4.1 Klassische Ergebnisse. Zunächst weist man mit verhältnismässig elementaren Mitteln nach, dass die nicht-trivialen Nullstellen von $\zeta(s)$ im kritischen Streifen $\{s \in \mathbb{C} \mid$ $0 \leq \operatorname{Re} s \leq 1\}$ liegen. Bezeichnet nun $N(T)$ die Anzahl der Nullstellen $s=\sigma+i$ im kritischen Streifen mit $0 \leq t \leq T$, so war bereits Riemann die Asymptotik

$$
N(T) \sim \frac{T}{2 \pi} \log \left(\frac{T}{2 \pi}\right) \quad(T \rightarrow \infty)
$$

bekannt, welche ihn zu seiner Vermutung führte, da er experimentell in etwa ebenso viele Nullstellen auf der kritischen Geraden fand. Einen wichtigen Beitrag zur Eingrenzung der Nullstellen innerhalb des kritischen Streifens gelang E. Littlewood im Jahr 1922; allerdings ist man damit noch weit von einem Beweis der Riemannschen Vermutung entfernt. In den Folgejahren wurden im Zuge der Verbesserung der Computertechnik vermehrt numerische Experimente zur Verifikation der Riemannschen Vermutung durchgeführt. In diesem Zusammenhang sind die eindrücklichen Ergebnisse von A. Odlyzko zu erwähnen, der gegenwärtig in der Grössenordnung von $10^{22}$ Nullstellen der Zetafunktion auf der kritischen Geraden berechnet hat (siehe [7]).

4.2 Ältere und neuere Bezüge. Die Riemannsche Vermutung hat E. Artin (1898-1962) und A. Weil (1906-1998) zu analogen Vermutungen zur Kongruenzzetafunktion algebraischer Varietäten über endlichen Körpern veranlasst. Beginnend mit den Resultaten von H. Hasse (1898-1979) in den 30er Jahren wurden diese Vermutungen in den 70er Jahren durch P. Deligne vollständig bewiesen, was als Evidenz für die Gültigkeit der Riemannschen Vermutung gewertet werden kann.

Die neuesten Entwicklungen zielen darauf ab, die Nullstellen von $\zeta(s)$ bzw. $\xi(t)$ als Eigenwerte eines unendlich dimensionalen Operators zu deuten, um dann die Riemannsche 
Vermutung mit Hilfe geeigneter kohomologischer Methoden (wie im Fall der Kongruenzzetafunktion) zu beweisen; diese Idee geht bereits auf D. Hilbert (1862-1943) zurück (siehe [4]). Eine weitere, überraschende Entdeckung von C. Deninger bringt die bereits erwähnten „Expliziten Formeln“ mit der Theorie gewisser dynamischer Systeme in Zusammenhang (siehe [5]). Schliesslich verweisen wir auf die ebenso überraschenden Verbindungen zur Physik, genauer zu chaotischen, quantenmechanischen Sytemen (siehe [3]).

\section{Literatur}

[1] Bombieri, E.: Problems of the Millennium: The Riemann Hypothesis. pdf-file unter http://www.claymath.org/prizeproblems/riemann.htm

[2] Chandrasekharan, K.: Arithmetical functions. Grundlehren der math. Wiss. 167. Springer-Verlag. Berlin, Heidelberg, New York 1970.

[3] Cipra, B.: A prime case of chaos. In: What's happening in the mathematical sciences. Vol. 4, AMS, 1999.

[4] Connes, A.: Trace formula in noncommutative geometry and the zeros of the Riemann zeta function. Selecta Math. 5 (1999), 29-106.

[5] Deninger, C.: Some analogies between number theory and dynamical systems on foliated spaces. Proc. Int. Congr. Math., vol. I, 163-186. Berlin 1998.

[6] Edwards, H.M.: Riemann's zeta function. Academic Press. New York, London 1974.

[7] Odlyzko, A.: Tables of zeros of the Riemann zeta function. http://www.dtc.umn.edu/ odlyzko/zeta_tables/index.html

[8] Riemann, B.: Gesammelte mathematische Werke, wissenschaftlicher Nachlass und Nachträge, Collected Papers. Springer-Verlag/B.G. Teubner Verlagsgesellschaft. Berlin, Heidelberg/Leipzig 1990.

[9] Zagier, D.: Newman's short proof of the prime number theorem. Am. Math. Mon. 104 (1997), 705-708.

[10] http://www.mersenne.org/prime.htm

Jürg Kramer

Institut für Mathematik

Humboldt-Universität zu Berlin

D-10099 Berlin

e-mail: kramer@mathematik.hu-berlin.de 